\title{
Trajectories of Maternal Depressive Symptoms From Pregnancy to First Six Months Postpartum and Child Neurodevelopment at Eight Months
}

\author{
Xiuxiu Li \\ Nanshan Maternity \& Child Healthcare Hospital of Shenzhen \\ Xuemei Liu \\ Nanshan Maternity \& Child Healthcare Hospital of Shenzhen \\ Min Wei \\ Nanshan Maternity \& Child Healthcare Hospital of Shenzhen \\ Xuhua Liu \\ Nanshan Maternity \& Child Healthcare Hospital of Shenzhen \\ Xiaojun Shi \\ Nanshan Maternity \& Child Healthcare Hospital of Shenzhen \\ Yanna Zhu \\ Sun Yat-sen University \\ Rui Gao ( $\nabla$ ruigao@szbcs.org ) \\ Nanshan Maternity \& Child Healthcare Hospital of Shenzhen
}

\section{Research Article}

Keywords: Trajectories, Maternal depression, Prenatal, Postpartum, Neurodevelopment

Posted Date: February 18th, 2022

DOI: https://doi.org/10.21203/rs.3.rs-1281796/v1

License: (9) (i) This work is licensed under a Creative Commons Attribution 4.0 International License. Read Full License 


\section{Abstract}

\section{Background:}

Maternal depression was an increasing risk of child neurodevelopment difficulties. However, maternal depression was always chronic and recurrent, and few studies have explored the association between the severity and duration of maternal depression and child development. We aimed to explore the relationship between trajectories of maternal depressive symptoms from pregnancy to the first six months postpartum and child development at eight months.

\section{Methods:}

Data was collected from 988 mother-child pairs who participated in Shenzhen Birth Cohort Study. Maternal depressive symptoms were evaluated by the Edinburgh Postnatal Depression Scale (EPDS) at four points: late pregnancy, 1 month, 3 months, and 6 months postpartum. Child emotional and behavioral developments were assessed by Ages and Stages Questionnaires: Social-Emotional (ASQ-SE) and Ages and Stages Questionnaires-Third Edition (ASQ-3) at 8 months postpartum. Latent profile class analysis (LPA) was used to identify the trajectories of maternal depressive symptoms. Univariate and multivariate linear regression were conducted to explore the association between the depressive symptoms trajectories and child development.

\section{Results:}

Four trajectories of maternal depressive symptoms were identified by LPA: Iow $(n=605)$, subclinical $(n=93)$, moderate-low and increasing $(n=255)$ and persistent high (54). Multivariable regressions showed that children of mothers assigned to persistent high depressive symptoms were more likely to have low scores in ASQ-3 total, three ASQ-3 domains (fine, problem-solving, and personal-society motor) and ASQ-se. Children of mothers assigned to class2 were more likely to have lower scores in ASQ-3 total, two ASQ-3 domains (communication motor and gross motor) and ASQ-se, compared to children of mothers assigned to class1. Risk factors identified in the multi-model included low birth weight, sleep quality at late pregnancy, low education level, and breastfeeding at 3 month.

\section{Conclusion:}

The impact of high levels of maternal depression symptoms on the increased risk of child developmental delay highlights the importance of early intervention and addressing maternal depression from pregnancy through early childhood. These findings draw attention to the need to move beyond a "depressed/not depressed" approach to screening for depression in the perinatal period.

\section{Introduction}

Child developmental delays, including communication, cognitive, social-emotional, gross, and fine motor [1, 2]. Delay in any one of the domains frequently results in the children lagging in a combination of the physical, cognitive, language, and socio-emotional developmental domains, which subsequently leads to adverse outcomes on children's health, academic achievement, and success later in their life[3]. More than 250 million (43\%) children under age 5 years in the low and middle-income countries could not achieve their full development potential[4]. Accumulating evidence has shown that maternal distress, including stress and depression, can have negative effects on child development in early life, which may ultimately influence lifelong health[5]. Additionally, women are particularly vulnerable to developing mental health problems during pregnancy and postpartum[6, 7]. About 13-21\% of prenatal women and 11$17 \%$ of postnatal women experience anxiety and depression $[8,9]$.

Existing literature has mainly focused on the impact of maternal distress on child development at a one-time point (i.e., at a single point in the prenatal or the postnatal period) $[10,11]$. However, recent studies suggested that maternal mental health symptoms are not isolated incidents and tend to recur or persist over time. For instance, a 2016 review focusing on longitudinal studies found that $39 \%$ of mothers who experienced prenatal developed to postpartum depression and $47 \%$ of women with postpartum depression also experienced prenatal depression[12]. A study suggests that $35.7 \%$ of women have persistently elevated symptoms over an 18-month period[13]. Similarly, $46.3 \%$ of the $16.9 \%$ of women with depression symptoms still had elevated symptoms at a 12-month follow-up assessment[14]. To date, few studies have explored the association between the severity and duration of maternal depression and child development. Trajectory analysis was a new tool using for describing the course of a measured variable over age or time. This method was originally developed for use in the fields of psychology and sociology, but has since been applied to a much wider range of fields, including epidemiology[15]. Some researchers have used this method to analyze long-term depression in mothers[16][17].

Given this, we aimed to identify different women groups by their trajectories of depressive symptoms across four points from late pregnancy to 6 months postpartum, and explore the associations between these trajectories and children's behavioral and emotional development at 8 months. The Latent Profile Analysis (LPA) approach was used to accessed the severity and persistence of maternal depression symptoms simultaneously[18, 19], which provides an opportunity to reduce a large heterogeneous group into several homogeneous subgroups, instead of employing the traditional cut-off approach.

\section{Methods}

\subsection{Participants}

This study is part of an ongoing birth cohort study, the Shenzhen Birth Cohort Study (SZBCS), which was conducted in Shenzhen Nanshan Maternity \& Child Healthcare Hospital of China. SZBCS is a population-based prospective cohort study, which designed to identify early environmental and genetic causes of normal and abnormal growth, development, and health from fetal life until young adulthood, SZBCS enrolled pregnant women before 19 weeks gestation from March 2018 and followed until young adulthood. Maternal and paternal information, clinical measures, and biological samples are collected at recruitment, 24-29, and 32-38 weeks of gestation. After delivery, neonatal birth information including mode of delivery and birth characteristics are obtained 
from routine medical records. For cohort children, Mothers are interviewed and children are examined in child health care clinics at age $1,3,6,12$ months, or by telephone interview for those mothers who are unable to bring their child to the pediatric clinic. The study plan to follow up all cohort children annually until age 18 years. Procedures and methods were approved by ethics committees at Nanshan Maternity \& Child Healthcare Hospital of Shenzhen (NSFYECKY-2020031), and informed consent was obtained from all participants.

This study included 1387 pregnant women enrolled in SZBCS who completed the prenatal questionnaires in the first, second, and third trimesters respectively. 1299 pregnant women agreed to participate after delivery. Of the 1299 mother-child pairs, 28 were excluded because they delivered twins, and one child because of congenital heart disease. Of the remaining 1271, 988 mother-child pairs finished the questionnaires at child one month, 3 months, 6 months, and 8 months, respectively. The main reasons for dropout were personal reasons, such as moving away, lack of interest, lack of time, except part of the child have not yet reached the survey months.

\subsection{Depression symptoms}

Depressive symptoms were accessed by the Edinburgh Postnatal Depression Scale (EPDS) [20]. The EPDS is a 10-item self-report instrument designed to measure the last 7days emotional expression during the postpartum period. Responses are scored from $0-3$, total scores with a range of $0-30$. A higher score suggested severer depression symptoms. Generally, a score of greater than 12 was recommended for identifying as depression. Good reliability and validity were demonstrated in the Chinese translation of EPDS, with internal consistency (Cronbach's a) of 0.87 [21]. The effectiveness of the scale in prenatal and postnatal has been recognized[22, 23]. In this study, the EPDS was asked to complete for mothers at 4-time points: late pregnancy, 1 month, 3 month, and 6month postpartum

\subsection{Emotional and behavioral development}

Child behavioral development was measured by the Ages and Stages Questionnaires-Third Edition (ASQ-3) [24] and social-emotional development was measured by the Ages and Stages Questionnaires: Social-Emotional (ASQ-SE) at ages 8 months. The ASQ-3/ASQ-SE is a parent-reported screening tool to capture the risk of developmental delays. ASQ-3 consists of 30 items, which were divided into 5 domains, namely communication, gross, fine, problemsolving, and personal-social. Each domain contains 6 items, and possible responses for each item were "yes" (10 points), "sometimes" (5 points), and "no" (0points). A total score of each domain is calculated as a sum of the 6 items ranging from 0 to 60 . The higher the score, the better the skills in the given domain. The ASQ-3 is a reliable and valid instrument to screen and monitor the development of Children in China, and the Cronbach's alpha coefficient was $0.8[25]$.

ASQ-SE consists of 8 versions depending on the different age groups, and the number of items ranges from 19 to 33 . There are three kinds of the answer: rarely or never, sometimes, most of the times, and 0,5 , and 10 were scored respectively. Additional 5 points were added If worrying about the child's performance of each item. The higher the total score, the worse social-emotional development. The internal consistency of the Chinese version's ASQ-se was measured by Cronbach's a, which ranged from 0.56-0.77 across various age intervals, and the item reliability ranged from 94-96\% [26].

\subsection{Covariates}

Information on Socio-demographic, obstetric conditions, and child feeding were collected by the follow-up questionnaires during pregnancy and postpartum. There are maternal age( $<25$ years vs. $25-34$ years vs. $\geq 35$ years $)$, pre-pregnancy BMI $\left(<18.5 \mathrm{~kg} / \mathrm{m}^{2} \mathrm{vs} .18 .5-23.9 \mathrm{~kg} / \mathrm{m}^{2} \mathrm{vs} .24-27.9 \mathrm{~kg} / \mathrm{m}^{2} \mathrm{vs} . \geq 28 \mathrm{~kg} / \mathrm{m}^{2}\right)$, a maternal education level (under and middle school vs. high school vs. college or university vs. post-graduated and higher), household residence type(Shenzhen residence vs. temporary residence vs. floating residence), parity(primigravida vs. multigravida), sleep quality at 3rd trimester(well vs. poor), maternal passive smoking(yes vs. no), Gestational diabetes mellitus(yes vs. no), subclinical hypothyroidism or hypothyroidism (yes vs. no), gestational hypertension (yes vs. no), breastfeeding at 3month(yes vs. no). Data of birth was collected from Shenzhen Maternity \& Child Healthcare hospital records, including birth weight and delivery gestational age. Low birth weight (LBW) was defined as birth weight less than 2500g, and preterm birth (PTB) was less than 37 weeks' gestation[27].

\subsection{Data statistics}

Statistical analysis was conducted using SPSS (23.0) and Mplus (7th ed)[28], and the $P$-value of less than 0.05 was considered to be statistically significant. Continuous variables were described as means $(X \pm S)$, and categorical variables as proportions $(n \%)$. Longitudinal profile class (LPA) was used to identify trajectories of women's depressive symptoms across four-time points (late pregnancy, 1, 3, and 6 months). To fit successive models, the number of classes was increased from 1, until the most parsimonious models with the smallest number of classes were identified. Model solutions were evaluated by comparing Likelihood ratio statistic (L2), Bayesian Information Criterion (BIC), and Akaike Information Criterion (AIC) across the successive models, Entropy is an index for assessing the precision of assigning latent class membership, with higher probability values indicating greater precisions of classification. The Vuong-Lo-Mendall-Rubin likelihood ratio test was also used to test for significant differences between the models[19]. The class membership of the most parsimonious model was saved and used in subsequent analysis. Then a bivariate linear regression analysis was used to measure the association between trajectories of maternal depression symptoms and child development (5 ASQ-3 domains, total scores, and social-emotional scores) at 8 months.

A multivariable linear regression model was used by adjusting confounders, including maternal age, pre-pregnancy BMI, maternal education level, household residence type, marital status, parity, sleep quality at 3rd trimester, maternal passive smoking, gestational diabetes mellitus, subclinical hypothyroidism, or hypothyroidism, gestational hypertension, breastfeeding at 3 month, LBW and PTB. Regression results were presented as adjusted b and $95 \%$ confidence intervals.

\section{Results}




\subsection{Sample characteristics}

A total of 988 mother-child pairs met all the study criteria and were included in this study finally. Table 1 describes the characteristics of the sample included in this study. Most of these women were between $25 \sim 34$ years of age at recruitment, were married, had a tertiary-level education, and had a temporary residence in Shenzhen in China. 526 (51\%) participants were primigravida. The proportion of women who reported EPDS scores $\geq 13$ (indicative of a probable diagnosis of major depression) in late pregnancy and at $1,3,6$ months postpartum was $6.0,7.8,12.9$, and $12.0 \%$, respectively.

Table 1

Characteristics of study participants

\begin{tabular}{|c|c|}
\hline Characteristics(N = 988) & $n(\%)$ \\
\hline \multicolumn{2}{|l|}{ Age } \\
\hline$<25$ & $27(2.7)$ \\
\hline $25 \sim 34$ & $680(68.8)$ \\
\hline$\geqq 35$ & $281(28.4)$ \\
\hline \multicolumn{2}{|l|}{ Pre-pregnancy BMI,(kg/m² ) } \\
\hline$<18.5$ & $159(16.1)$ \\
\hline $18.5 \sim 23.9$ & $667(67.5)$ \\
\hline $24 \sim 27.9$ & $100(10.1)$ \\
\hline$\geqq 28$ & $62(6.3)$ \\
\hline \multicolumn{2}{|l|}{ Education } \\
\hline Under and middle school & $43(4.4)$ \\
\hline high school & $97(9.8)$ \\
\hline College or University & $735(74.4)$ \\
\hline Post-graduated and higher & $113(11.4)$ \\
\hline \multicolumn{2}{|l|}{ Household registration type } \\
\hline Shenzhen residence & $627(61.5)$ \\
\hline Temporary residence & $378(37.1)$ \\
\hline Floating residence & $14(1.4)$ \\
\hline \multicolumn{2}{|l|}{ Marital status } \\
\hline unmarried & $29(2.9)$ \\
\hline married & $926(93.7)$ \\
\hline divorce & $33(3.3)$ \\
\hline \multicolumn{2}{|l|}{ Parity } \\
\hline Primigravida & $542(54.9)$ \\
\hline Multigravida & $446(44.3)$ \\
\hline \multicolumn{2}{|l|}{ Sleep quality at 3rd trimester } \\
\hline Well & $648(65.6)$ \\
\hline Poor & $340(34.4)$ \\
\hline Passive smoking, yes & $236(23.9)$ \\
\hline Late-pregnancy EPDS > 12 & $59(6.0)$ \\
\hline 1month postpartum EPDS $>12$ & $77(7.8)$ \\
\hline 3month postpartum EPDS $>12$ & $127(12.9)$ \\
\hline 6month postpartum EPDS $>12$ & $118(12.0)$ \\
\hline
\end{tabular}

\subsection{Trajectories of maternal depressive symptoms}

The final model with four trajectories of maternal depressive symptoms, at four-time points (late pregnancy, 1, 3, and 6 months postpartum) were accepted, as its fit indexes(entropy $=0.822$ ) were higher than the 2 - and 3-class models (supplemental table). Additionally, the entropy value for the 4 -class model was 
highest(entropy $=0.822)$, suggesting the best precision in assigning the individual to their classes. The 3-class model was not chosen, despite a significant result from the Vuong-Lo-Mendell-Rubin likelihood ratio test and LMR due to its lower entropy than the 4-class model[29]. Figure 1 shows the 4-class trajectories of maternal depression symptoms. The first trajectory characterized by persistent low levels of depressive symptoms, consisted of $60.1 \%(n=$

605 ) of mothers. The second trajectory presented a pattern of subclinical depressive symptoms with a high depressive symptom score at pregnancy, and had a decrease at postpartum, comprising $9.2 \%(n=93)$ of mothers. The third trajectory consists of $25.3 \%(n=255)$ mothers, who presented a pattern of moderate depressive symptoms during pregnancy with increasing symptoms in postpartum. The fourth group included $5.4 \%(n=54)$ of the mothers, who were characterized by persistently high levels of depressive symptoms. Therefore, the 1- to 4-class trajectories of depressive symptoms were named orderly as "low depressive", "subclinical depressive", "moderate-low and increasing" and "persistent high depressive".

\subsection{Associations between trajectories of maternal depressive symptom and child emotional and behavioral development}

The associations between maternal depression symptom trajectories and 5 ASQ-3 domains (communication motor, gross motor, fine motor, problem-solving motor, and personal-society motor), ASQ-3 total score, and ASQ-se score are presented in Table2-4. Covariates were adjusted in multivariable analysis, including maternal age, pre-pregnancy BMI, maternal education, sleep quality at late-pregnancy, passive smoking antenatal, parity, GDM, subclinical hypothyroidism or hypothyroidism, low birth weight, preterm birth, and breastfeeding at 3month. At eight months, the ASQ-3 total score of children of persistent high depressive symptom group had an increased risk of low score of ASQ-3 than children of mother in other groups, as were their ASQ-se score and 3 ASQ-3 domains (fine motor, problem-solving, and personal-society), even after adjusting for confounding. Furthermore, the final multivariable model showed that children of mothers assigned to class2 were more likely to have lower scores in ASQ-3 total, 2 ASQ-3 domains (communication motor and gross motor), and ASQ-se, compared with children of class1, after adjusting for covariates. Risk factors identified in the multi-model included low birth weight, sleep quality at late pregnancy, low education, and breastfeeding at 3month. 
Table 2

Univariate and multivariable linear regression model predicting children's communication, gross and fine motor difficulties at 8 months

\begin{tabular}{|c|c|c|c|c|c|c|}
\hline & \multicolumn{2}{|c|}{ Communication } & \multicolumn{2}{|l|}{ Gross motor } & \multicolumn{2}{|l|}{ Fine motor } \\
\hline & Model1 ${ }^{a}$ & Model2 ${ }^{b}$ & Model1 a & Model2 ${ }^{b}$ & Model1 a & Model2 ${ }^{b}$ \\
\hline & $\beta(95 \% C l)$ & $\beta(95 \% \mathrm{Cl})$ & $\beta(95 \% C l)$ & $\beta(95 \% C l)$ & $\beta(95 \% C l)$ & $\beta(95 \% C l)$ \\
\hline \multicolumn{7}{|l|}{ Depressive symptoms trajectories } \\
\hline Low depressive & Reference & Reference & Reference & Reference & Reference & Reference \\
\hline Subclinical depressive & $\begin{array}{l}-2.53(-4.29 \\
-0.78)\end{array}$ & $\begin{array}{l}-2.48(-4.32 \\
-0.64)\end{array}$ & $\begin{array}{l}-2.35(-4.2 \\
-0.51)\end{array}$ & $\begin{array}{l}-2.17(-4.09 \\
-0.25)\end{array}$ & $\begin{array}{l}-0.69(-2.20 \\
0.82)\end{array}$ & $-0.5(-2.08,1.07)$ \\
\hline Moderate-low and increasing depressive & $\begin{array}{l}-0.62(-1.80 \\
0.56)\end{array}$ & $\begin{array}{l}-0.71(-1.94 \\
0.52)\end{array}$ & $\begin{array}{l}-0.39(-1.63 \\
0.85)\end{array}$ & $-0.26(-1.55,1.02)$ & $\begin{array}{l}-0.93(1.94 \\
0.09)\end{array}$ & $\begin{array}{l}-0.72(-1.77 \\
0.33)\end{array}$ \\
\hline Persistent high depressive & $\begin{array}{l}-2.63(-4.89 \\
-0.37)\end{array}$ & $\begin{array}{l}-2.26(-4.62 \\
0.10)\end{array}$ & $\begin{array}{l}-1.99(-4.36 \\
0.39)\end{array}$ & $-1.31(-3.78,1.16)$ & $\begin{array}{l}-2.80(-4.75 \\
-0.86)\end{array}$ & $-2.3(-4.32,-0.29)$ \\
\hline \multicolumn{7}{|l|}{ Covariates } \\
\hline $\mathrm{BMI}\left(\geqq 28 \mathrm{~kg} / \mathrm{m}^{2}\right)$ & & \multicolumn{2}{|l|}{$-0.14(-3.32,3.03)$} & \multicolumn{2}{|l|}{$-0.48(-3.80,2.84)$} & $-0.27(-2.99,2.44)$ \\
\hline $\mathrm{BMI}\left(24 \sim 27.9 \mathrm{~kg} / \mathrm{m}^{2}\right)$ & & \multicolumn{2}{|l|}{$-0.61(-2.70,1.47)$} & \multicolumn{2}{|l|}{$-1.12(-3.31,1.06)$} & $-0.93(-2.71,0.85)$ \\
\hline BMI $\left(18.5 \sim 23.9 \mathrm{~kg} / \mathrm{m}^{2}\right)$ & & \multicolumn{2}{|l|}{$0.26(-1.17,1.70)$} & \multicolumn{2}{|l|}{$0.05(-1.45,1.55)$} & $-0.04(-1.27,1.18)$ \\
\hline Maternal age(>35) & & \multicolumn{2}{|l|}{$0.76(-2.63,4.15)$} & \multicolumn{2}{|l|}{$1.19(-2.35,4.74)$} & $-0.72(-3.61,2.18)$ \\
\hline Maternal age(25 34) & & \multicolumn{2}{|l|}{$0.71(-2.47,3.90)$} & \multicolumn{2}{|l|}{$1.76(-1.58,5.10)$} & $-0.40(-3.13,2.32)$ \\
\hline Primigravida(yes) & & \multicolumn{2}{|l|}{$0.6(-0.55,1.75)$} & \multicolumn{2}{|l|}{$0.67(-0.54,1.87)$} & $0.41(-0.57,1.4)$ \\
\hline Maternal passive smoking(yes) & & \multicolumn{2}{|l|}{$0.16(-1.15,1.47)$} & \multicolumn{2}{|l|}{$0.05(-1.33,1.42)$} & $0.58(-0.54,1.7)$ \\
\hline Preterm(yes) & & \multicolumn{2}{|l|}{$-0.63(-3.46,2.20)$} & \multicolumn{2}{|l|}{$1.05(-1.91,4.01)$} & $0.11(-2.31,2.53)$ \\
\hline Gestational hypertension(yes) & & \multicolumn{2}{|l|}{$0.94(-1.14,3.03)$} & \multicolumn{2}{|l|}{$0.33(-1.86,2.51)$} & $0.78(-1.01,2.56)$ \\
\hline Fetus gender(girl) & & \multicolumn{2}{|l|}{$1.27(0.24,2.30)$} & \multicolumn{2}{|l|}{$-0.81(-1.89,0.27)$} & $0.91(0.03,1.79)$ \\
\hline $\begin{array}{l}\text { Subclinical hypothyroidism or } \\
\text { hypothyroidism (yes) }\end{array}$ & & \multicolumn{2}{|l|}{$0.00(-1.65,1.65)$} & \multicolumn{2}{|l|}{$-0.72(-2.44,1.01)$} & $-0.86(-2.27,0.55)$ \\
\hline Gestational diabetes mellitus(yes) & & \multicolumn{2}{|l|}{$-0.18(-1.54,1.19)$} & \multicolumn{2}{|l|}{$0.71(-0.72,2.14)$} & $0.22(-0.95,1.39)$ \\
\hline Breastfeeding at 3months & & \multicolumn{2}{|l|}{$1.41(-0.37,3.19)$} & $2.61(0.74,4.48)$ & & $0.37(-1.16,1.90)$ \\
\hline Low birth weight(yes) & & $-0.61(-3.92,2.71)$ & & $\begin{array}{l}-3.66(-7.13 \\
-0.19)\end{array}$ & & $\begin{array}{l}-4.35(-7.19 \\
-1.51)\end{array}$ \\
\hline Sleep quality at late-pregnancy(poor) & & $-0.12(-1.23,0.99)$ & & $-0.24(-1.41,0.92)$ & & $\begin{array}{l}-1.05(-2.00 \\
-0.10)\end{array}$ \\
\hline Education (high school) & & $1.96(-0.91,4.82)$ & & $2.41(-0.59,5.41)$ & & $1.46(-0.99,3.91)$ \\
\hline Education (College or University) & & $0.66(-1.91,3.22)$ & & $0.96(-1.72,3.64)$ & & $0.93(-1.26,3.12)$ \\
\hline Education (Post-graduated and higher) & & $0.74(-2.25,3.73)$ & & $2.22(-0.91,5.35)$ & & $1.19(-1.37,3.74)$ \\
\hline Residence type (Temporary residence) & & $-0.13(-1.33,1.07)$ & & $-0.42(-1.67,0.84)$ & & $0.28(-0.74,1.31)$ \\
\hline Residence type (Floating residence) & & $1.18(-2.19,4.55)$ & & $2.17(-1.35,5.70)$ & & $0.58(-2.03,3.46)$ \\
\hline *[a]Model1 was not adjusted by previous & undings. & & & & & \\
\hline $\begin{array}{l}\text { *[b]Model } 2 \text { was adjusted for potential cor } \\
\text { gestational hypertension, fetus gender, su } \\
\text { pregnancy and residence type. }\end{array}$ & $\begin{array}{l}\text { ding variabl } \\
\text { cal hypothy }\end{array}$ & $\begin{array}{l}\text { luding pre-pregna } \\
\text { m, or hypothyroid }\end{array}$ & $\begin{array}{l}\text { y BMI, mater } \\
\text { h, breastfeed }\end{array}$ & $\begin{array}{l}\text { age, parity, mater } \\
\text { at } 3 \text { months, low }\end{array}$ & $\begin{array}{l}\text { passive sm } \\
\text { h weight sl }\end{array}$ & $\begin{array}{l}\text { gg, preterm, } \\
\text { quality at late- }\end{array}$ \\
\hline
\end{tabular}


Table 3

Univariate and multivariable linear regression model predicting children's problem solving, personal-society motor, and ASQ-3total difficulties at 8months

Problem motor

Model $1^{\mathrm{a}} \quad$ Model $2^{\mathrm{b}}$
Personal-society motor

Model $1^{\mathrm{a}} \quad$ Model $2^{\mathrm{b}}$

\section{Total score}

Model1 ${ }^{a} \quad$ Model $2^{b}$ $\beta(95 \% \mathrm{Cl}) \quad \beta(95 \% \mathrm{Cl}) \quad \beta(95 \% \mathrm{Cl}) \quad \beta(95 \% \mathrm{Cl}) \quad \beta(95 \% \mathrm{Cl}) \quad \beta(95 \% \mathrm{Cl})$

Depressive symptoms trajectories

Low depressive

Subclinical depressive

Reference

$-0.64(-2.19,0.92) \quad-0.57(-2.2,1.06)$

Reference

$-1.26(-3.06,0.55)$

$-0.88(-1.92,0.17)$

Moderate-low and increasing

depressive

Persistent high depressive

$-3.82(-5.82$ $-1.82)$

Covariates

BMI $\left(\geqq 28 \mathrm{~kg} / \mathrm{m}^{2}\right)$

$\operatorname{BMI}\left(18.5 \sim 23.9 \mathrm{~kg} / \mathrm{m}^{2}\right)$

Maternal age(>35)

Maternal age(25 34)

Primigravida(yes)

Maternal passive smoking(yes)

Preterm(yes)

Gestational hypertension(yes)

Fetus gender(girl)

Subclinical hypothyroidism or

hypothyroidism (yes)

Gestational diabetes mellitus(yes)

Breastfeeding at 3months

Low birth weight(yes)

Sleep quality at late-

pregnancy(poor)

Education (high school)

Education (College or University)

Education (Post-graduated and higher)

Residence type (Temporary

residence)

Residence type (Floating

residence)
$\operatorname{BMI}\left(24 \sim 27.9 \mathrm{~kg} / \mathrm{m}^{2}\right)$

$-0.49(-3.31,2.33)$

$-0.57(-2.42,1.28)$

$0.14(-1.13,1.42)$

$-0.65(-3.66,2.36)$

$-0.82(-3.66,2.01)$

$0.36(-0.66,1.38)$

$0.36(-0.8,1.53)$

$0.78(-1.73,3.3)$

$0.61(-1.25,2.46)$

$-0.14(-1.05,0.78)$

$-0.6(-2.07,0.86)$

$-0.22(-1.44,0.99)$

$0.68(-0.91,2.26)$

$-0.61(-3.56,2.34)$

$0.13(-0.86,1.12)$

$1.5(-1.05,4.04)$

$1.65(-0.63,3.93)$

$2.8(0.14,5.46)$

$0.44(-0.62,1.51)$

$2.19(-0.8,5.19)$

*[a]Model1 was not adjusted by previous confoundings

*[b]Model2 was adjusted for potential confounding variables including pre-pregnancy BMI, maternal age, parity, maternal passive smoking, preterm, gestational hypertension, fetus gender, subclinical hypothyroidism, or hypothyroidism, breastfeeding at 3 months, low birth weight, sleep quality at latepregnancy and residence type. 


\begin{tabular}{|c|c|c|}
\hline & \multicolumn{2}{|l|}{ ASQ-se } \\
\hline & Model $1^{\mathrm{a}}$ & Model $2^{b}$ \\
\hline & $\beta(95 \% \mathrm{Cl})$ & $\beta(95 \% \mathrm{Cl})$ \\
\hline \multicolumn{3}{|l|}{ Depressive symptoms trajectories } \\
\hline Low depressive & Reference & Reference \\
\hline Subclinical depressive & $4.31(0.94,7.68)$ & $4.12(0.65,7.58)$ \\
\hline Moderate-low and increasing depressive & $5.06(2.8,7.31)$ & $4.86(2.54,7.18)$ \\
\hline Persistent high depressive & $11.11(6.81,15.4)$ & $9.49(5.09,13.9)$ \\
\hline \multicolumn{3}{|l|}{ Covariates } \\
\hline \multicolumn{2}{|l|}{ Pre-pregnancy BMI ( $\geqq 28$ kg/m²) } & $-5.24(-11.22,0.75)$ \\
\hline \multicolumn{2}{|l|}{ Pre-pregnancy BMI $(24 \sim 27.9$ kg/m²) } & $1.14(-2.79,5.08)$ \\
\hline \multicolumn{2}{|l|}{ Pre-pregnancy BMI $(18.5 \sim 23.9$ kg/m²) } & $0.85(-1.85,3.56)$ \\
\hline \multicolumn{2}{|l|}{ Maternal age $(>35)$} & $-3.55(-9.94,2.84)$ \\
\hline \multicolumn{2}{|l|}{ Maternal age(25 34$)$} & $-1.39(-7.4,4.63)$ \\
\hline \multicolumn{2}{|l|}{ Primigravida(yes) } & $-0.52(-2.69,1.65)$ \\
\hline \multicolumn{2}{|l|}{ Maternal passive smoking(yes) } & $1.36(-1.12,3.83)$ \\
\hline \multicolumn{2}{|l|}{ Preterm(yes) } & $2.87(-2.46,8.21)$ \\
\hline \multicolumn{2}{|l|}{ Gestational hypertension(yes) } & $2.7(-1.22,6.61)$ \\
\hline \multicolumn{2}{|l|}{ Fetus gender(girl) } & $1.33(-0.61,3.28)$ \\
\hline \multicolumn{2}{|l|}{ Subclinical hypothyroidism or hypothyroidism (yes) } & $0.47(-2.64,3.58)$ \\
\hline \multicolumn{2}{|l|}{ Gestational diabetes mellitus (yes) } & $2.23(-0.35,4.8)$ \\
\hline \multicolumn{2}{|l|}{ Breastfeeding at 3months } & $-1.19(-4.56,2.17)$ \\
\hline \multicolumn{2}{|l|}{ Low birth weight(yes) } & $-3.06(-9.32,3.2)$ \\
\hline \multicolumn{2}{|l|}{ Sleep quality at late-pregnancy(poor) } & $2.11(0.02,4.21)$ \\
\hline \multicolumn{2}{|l|}{ Education (high school) } & $-5.23(-10.64,0.17)$ \\
\hline \multicolumn{2}{|l|}{ Education (College or University) } & $-6.94(-11.78,-2.1)$ \\
\hline \multicolumn{2}{|l|}{ Education (Post-graduated and higher) } & $-8.36(-14,-2.72)$ \\
\hline \multicolumn{2}{|l|}{ Residence type (Temporary residence) } & $0.21(-2.06,2.47)$ \\
\hline \multicolumn{2}{|l|}{ Residence type (Floating residence) } & $-2.97(-9.32,3.38)$ \\
\hline \multicolumn{3}{|l|}{ *[a]Model1 was not adjusted by previous confoundings } \\
\hline \multicolumn{3}{|c|}{$\begin{array}{l}\text { *[b]Model2 was adjusted for potential confounding variables including pre-pregnancy BMI, maternal age, parity, maternal passive smoking, preterm, } \\
\text { gestational hypertension, fetus gender, subclinical hypothyroidism, or hypothyroidism, breastfeeding at 3months, low birth weight, sleep quality at late- } \\
\text { pregnancy and residence type. }\end{array}$} \\
\hline
\end{tabular}

\section{Discussion}

The present study identified four groups of maternal depressive symptoms trajectories, from late pregnancy to 6 months after birth: "low depressive", "subclinical depressive", "moderate-low and increasing" and "persistent high depressive". The influence of severity and chronicity of maternal depressive symptoms on child development has been examined. We found that mothers experiencing persistent high symptoms are more likely to report that their children have a lower score on the ASQ-3 total, ASQ-se, and 3 ASQ-3 domains (fine, problem-solving, and personal-society motor). Similarly, children of mothers assigned to subclinical depressive symptoms were more likely to have lower scores in ASQ-3 total, 2 ASQ-3 domains (communication motor and gross motor), and ASQ-se, compared with children of low depressive symptoms. This study investigated the effect of maternal depression symptoms over time and found that "subclinical symptoms" and "persistent high symptoms" had an increased risk for poor emotional and behavioral development. As such, this study provides novel evidence of the negative impact of persistently high and subclinical depressive symptoms on delayed child development at eight months. 
Some previous research supports our view. An association between maternal depressive symptoms at prenatal or postnatal, and child poor neurodevelopment was found [10,11]. Kingston et al. explored the depression symptoms across four points from mid-pregnancy to one year postpartum and identified four distinct trajectories of maternal depressive symptoms: low level (64.7\%), early postpartum (10.9\%), subclinical (18.8\%), and persistent high (5.6\%). In multivariable models, the children born to women with persistent high depressive symptoms had the highest proportion of elevated behavior symptoms, followed by mothers with moderate symptoms (early postpartum and subclinical trajectories) and lowest for minimal symptoms[30]. Another $\operatorname{study}(\mathrm{N}=1983)$ also supporting mothers experiencing high anxiety symptoms in the perinatal period have an adverse impact on child developmental delays. They identified three distinct trajectories of maternal stress and anxiety symptoms from mid-pregnancy to 3 years postpartum. Multivariate analysis showed mothers belonging to the high anxiety symptoms class had an increased risk (adjusted OR $2.80,95 \% \mathrm{Cl} 2.80(1.42-5.51)$, p $=0.003)$ of having a child with developmental delays at 3 years[31]. But their explorations are limited to one aspect of child development, caused by maternal depression. As a result, they are unable to capture the simultaneous influence of maternal depression on multiple areas of child development, such as communication, motor, problemsolving and social-emotional development. It is important to note that, maternal with high depressive symptoms at prenatal had a significant effect on poor emotional and behavioral development compared with increased and persistent even at low-level symptoms. Researchers found that children of mothers who had elevated depressive symptoms during pregnancy had worse mental health in adolescence[32, 33]. The effect of prenatal maternal depressive symptoms on child outcomes is noteworthy. Further studies are warranted to explore how the course of prenatal and postnatal depression affects child development.

In the final multivariate model, we explored the relationships between maternal depression symptom trajectories and children's emotional and behavioral development after accounting for demographic, obstetrics, birth outcomes, and feeding factors. sleep quality at late pregnancy, low birth weight, breastfeeding at 3 month and lower education level of mothers were also associated with poorer emotional and behavioral for development children. Some of the characteristics might be pathways by which maternal mental health difficulties contribute to children's emotional and behavioral difficulties. For instance, depression symptom might lead to chronic sleep loss and low birth weight. The former might contribute to adverse pregnancy outcomes and maternal and child stress 'overload', and the latter might increase the gross and fine motors risk of a diagnosis of neurodevelopment delay[34]. Depression symptom can also affect children by affecting mothers' behavior. Studies showed positive associations between breastfeeding and child neurodevelopment[35, 36].

Our findings have implications for physicians who provide care during pregnancy. Clinically, if we only considered women's scores using the recommended screening cut-off of 13 or greater on the EPDS, those assigned to the subclinical depressive symptom trajectory may not be identified as their mean scores are below the clinical cut-off points. However, the proportion of subclinical women is high, and the significant effect on the child neurodevelopment, this raises questions for current health care that rely on cut-offs to evaluate women's mental health requiring intervention or follow up, and provide evidence of early identification and support.

This study benefits from using longitudinal statistical modeling of data drawn from a prospective birth cohort study. However, there are several limitations to note. First, The EPDS test was self-written by the mother, which might lead self-report bias. Second, the EPDS was only assessed at late pregnancy for all women, not at first and second trimester, preventing classifying the trajectory of depressive symptoms during pregnancy by trimester. Third, we couldn't include all covariates associated with children's outcomes $\mathrm{n}$ the study. Finally, we supposed data were missing at random in the present analysis, this may result in an underestimate of maternal depressive symptoms as more depressed women may be more likely to miss a study assessment.

\section{Conclusion}

Women's depressive symptoms from late pregnancy through postpartum at six months followed four distinct trajectories defined by low-level symptoms, increase and persistent ant low-level symptoms, subclinical depressive symptoms, and persistent high symptoms. Even after accounting for demographic, obstetric, birth outcomes, and feeding factors, the association between depression symptom trajectories and child emotional and behavioral development remained significant. Especially sub-clinical levels of depressive symptoms are associated with adverse emotional-behavioral outcomes for children. These findings draw attention to the need to move beyond a "depressed/not depressed" approach to screening for depression in the perinatal period. And the findings of the present study have important implications for policy and practice efforts focused on improving maternal and child health in the perinatal and early childhood period.

\section{Declarations}

\section{Ethics approval and consent to participate}

Procedures and methods were approved by ethics committees at Nanshan Maternity \& Child Healthcare Hospital of Shenzhen (NSFYEC-KY-2020031), and informed consent was obtained from all participants or their legal guardians.

\section{Methods}

We confirm that all methods were performed in accordance with the relevant guidelines and regulations.

\section{Consent for publication}

Not applicable.

\section{Availability of data and materials}


The datasets that support the findings of this study are available on reasonable request from the corresponding author. The data are not publicly available due to privacy or ethical restrictions.

\section{Competing Interest}

The authors have no conflict of interest to declare.

\section{Funding Source}

This study was sponsored by the Medical Scientific Research Foundation of Guangdong Province of China (A2021123), the Science and Technology Planning Project of ShenZhen Nanshan District (2020032 General), and the Sanming Project of Medicine in Shenzhen (SZSM201803061).

\section{Acknowledgments}

We are thankful to the families taking part in the study and the members of the Shenzhen Birth Cohort Study team, who assisted with data collection.

\section{Author's contributions}

XXL and RG conceived and designed the study. XXL conducted the statistical analysis and drafted the manuscript. RG supervised the study and provided administrative, technical, and material support. All authors critically reviewed the manuscript and approved the final version submitted for publication.

\section{References}

1. Montes G, Lotyczewski BS, Halterman JS, Hightower AD: School readiness among children with behavior problems at entrance into kindergarten: results from a US national study. EUR J PEDIATR 2012, 171(3):541-548.

2. Obradovic J, Burt KB, Masten AS: Testing a dual cascade model linking competence and symptoms over 20 years from childhood to adulthood. $\mathrm{J}$ Clin Child Adolesc Psychol 2010, 39(1):90-102.

3. Blair C, Raver CC: Child development in the context of adversity: experiential canalization of brain and behavior. AM PSYCHOL 2012, 67(4):309-318.

4. WHO: WHO. WWW Document http://www.who.int/topics/early-child-development/en/. In.; 2016.

5. Liu Y, Kaaya S, Chai J, McCoy DC, Surkan PJ, Black MM, Sutter-Dallay AL, Verdoux H, Smith-Fawzi MC: Maternal depressive symptoms and early childhood cognitive development: a meta-analysis. PSYCHOL MED 2017, 47(4):680-689.

6. Austin MP: Antenatal screening and early intervention for "perinatal" distress, depression and anxiety: where to from here? Arch Womens Ment Health 2004, 7(1):1-6.

7. Highet N, Stevenson AL, Purtell C, Coo S: Qualitative insights into women's personal experiences of perinatal depression and anxiety. WOMEN BIRTH 2014, 27(3):179-184.

8. Dennis CL, Falah-Hassani K, Shiri R: Prevalence of antenatal and postnatal anxiety: systematic review and meta-analysis. Br J Psychiatry 2017, 210(5):315-323.

9. Fairbrother N, Young AH, Janssen P, Antony MM, Tucker E: Depression and anxiety during the perinatal period. BMC PSYCHIATRY 2015, 15:206.

10. Oyetunji A, Chandra P: Postpartum stress and infant outcome: A review of current literature. Psychiatry Res 2020, 284:112769.

11. Ghimire U, Papabathini SS, Kawuki J, Obore N, Musa TH: Depression during pregnancy and the risk of low birth weight, preterm birth and intrauterine growth restriction- an updated meta-analysis. EARLY HUM DEV 2021, 152:105243.

12. Underwood L, Waldie K, D'Souza S, Peterson ER, Morton S: A review of longitudinal studies on antenatal and postnatal depression. Arch Womens Ment Health 2016, 19(5):711-720.

13. McLennan JD, Kotelchuck M, Cho H: Prevalence, persistence, and correlates of depressive symptoms in a national sample of mothers of toddlers. J Am Acad Child Adolesc Psychiatry 2001, 40(11):1316-1323.

14. Horwitz SM, Briggs-Gowan MJ, Storfer-Isser A, Carter AS: Prevalence, correlates, and persistence of maternal depression. J Womens Health (Larchmt) 2007, 16(5):678-691.

15. Nguena NH, Pagé MG, Katz J, Choinière M, Vanasse A, Dorais M, Samb OM, Lacasse A: Trajectory Modelling Techniques Useful to Epidemiological Research: A Comparative Narrative Review of Approaches. CLIN EPIDEMIOL 2020, 12:1205-1222.

16. Jacques N, Mesenburg MA, Matijasevich A, Domingues MR, Bertoldi AD, Stein A, Silveira MF: Trajectories of maternal depressive symptoms from the antenatal period to 24-months postnatal follow-up: findings from the 2015 Pelotas birth cohort. BMC PSYCHIATRY 2020, 20 (1):233.

17. Kingston D, Kehler H, Austin MP, Mughal MK, Wajid A, Vermeyden L, Benzies K, Brown S, Stuart S, Giallo R: Trajectories of matemal depressive symptoms during pregnancy and the first 12 months postpartum and child externalizing and internalizing behavior at three years. PLOS ONE 2018, $13(4)$ :e195365.

18. Cents RA, Diamantopoulou S, Hudziak JJ, Jaddoe VW, Hofman A, Verhulst FC, Lambregtse-van DBM, Tiemeier H: Trajectories of maternal depressive symptoms predict child problem behaviour: the Generation R study. PSYCHOL MED 2013, 43(1):13-25.

19. Lubke G, Neale MC: Distinguishing Between Latent Classes and Continuous Factors: Resolution by Maximum Likelihood? Multivariate Behav Res 2006, 41(4):499-532.

20. Cox JL, Holden JM, Sagovsky R: Detection of postnatal depression. Development of the 10-item Edinburgh Postnatal Depression Scale. Br J Psychiatry 1987, 150:782-786. 
21. Szegda K, Markenson G, Bertone-Johnson ER, Chasan-Taber L: Depression during pregnancy: a risk factor for adverse neonatal outcomes? A critical review of the literature. J Matern Fetal Neonatal Med 2014, 27(9):960-967.

22. Talge NM, Neal C, Glover V: Antenatal maternal stress and long-term effects on child neurodevelopment: how and why? J Child Psychol Psychiatry 2007, 48(3-4):245-261.

23. Gavin NI, Gaynes BN, Lohr KN, Meltzer-Brody S, Gartlehner G, Swinson T: Perinatal depression: a systematic review of prevalence and incidence. OBSTET GYNECOL 2005, 106(5 Pt 1):1071-1083.

24. Squires JD: Ages \& Stages Questionnaires[R], Third Edition (ASQ-3[TM]): A Parent-Completed Child-Monitoring System. Brookes Publishing Company 2009.

25. Wei M, Bian X, Squires J, Yao G, Wang X, Xie H, Song W, Lu J, Zhu C, Yue H et al: [Studies of the norm and psychometrical properties of the ages and stages questionnaires, third edition, with a Chinese national sample]. Zhonghua Er Ke Za Zhi 2015, 53(12):913-918.

26. Bian X, Xie H, Squires J, Chen CY: ADAPTING A PARENT-COMPLETED, SOCIOEMOTIONAL QUESTIONNAIRE IN CHINA: THE AGES \& STAGES QUESTIONNAIRES: SOCIAL-EMOTIONAL. INFANT MENT HEALTH J 2017, 38(2):258-266.

27. Shen ZZ, Wang YW, Ma S, Zhan YL, Wu SS, Feng YH, Cai SY, Ma LK, Jiang Y: [Risk factors for preterm birth, low birth weight and small for gestational age: a prospective cohort study]. Zhonghua Liu Xing Bing Xue Za Zhi 2019, 40(9):1125-1129.

28. Muthén L, Muthén B: Mplus user's guide (7th ed.). 2012.

29. Park M, Brain U, Grunau RE, Diamond A, Oberlander TF: Maternal depression trajectories from pregnancy to 3 years postpartum are associated with children's behavior and executive functions at 3 and 6 years. Arch Womens Ment Health 2018, 21(3):353-363.

30. Kingston D, Kehler H, Austin MP, Mughal MK, Wajid A, Vermeyden L, Benzies K, Brown S, Stuart S, Giallo R: Trajectories of matemal depressive symptoms during pregnancy and the first 12 months postpartum and child externalizing and internalizing behavior at three years. PLOS ONE 2018, $13(4)$ :e195365.

31. Mughal MK, Giallo R, Arnold P, Benzies K, Kehler H, Bright K, Kingston D: Trajectories of maternal stress and anxiety from pregnancy to three years and child development at 3 years of age: Findings from the All Our Families (AOF) pregnancy cohort. J Affect Disord 2018, 234:318-326.

32. Ed. KSBM, Sc. GMWB, Najman JM, Dip. RAMA: MATERNAL DEPRESSIVE, ANXIOUS, AND STRESS SYMPTOMS DURING PREGNANCY PREDICT INTERNALIZING PROBLEMS IN ADOLESCENCE. Depression \& Anxiety 2014, 31(1):9-18.

33. Betts KS, Williams GM, Najman JM, Alati R: THE RELATIONSHIP BETWEEN MATERNAL DEPRESSIVE, ANXIOUS, AND STRESS SYMPTOMS DURING PREGNANCY AND ADULT OFFSPRING BEHAVIORAL AND EMOTIONAL PROBLEMS. Depression \& Anxiety 2014, 32(2):82-90.

34. Palagini L, Gemignani A, Banti S, Manconi M, Mauri M, Riemann D: Chronic sleep loss during pregnancy as a determinant of stress: impact on pregnancy outcome. SLEEP MED 2014, 15(8):853-859.

35. Belfort MB, Rifas-Shiman SL, Kleinman KP, Guthrie LB, Bellinger DC, Taveras EM, Gillman MW, Oken E: Infant feeding and childhood cognition at ages 3 and 7 years: Effects of breastfeeding duration and exclusivity. JAMA PEDIATR 2013, 167(9):836-844.

36. Huang J, Peters KE, Vaughn MG, Witko C: Breastfeeding and trajectories of children's cognitive development. Dev Sci 2014, 17(3):452-461.

\section{Figures}

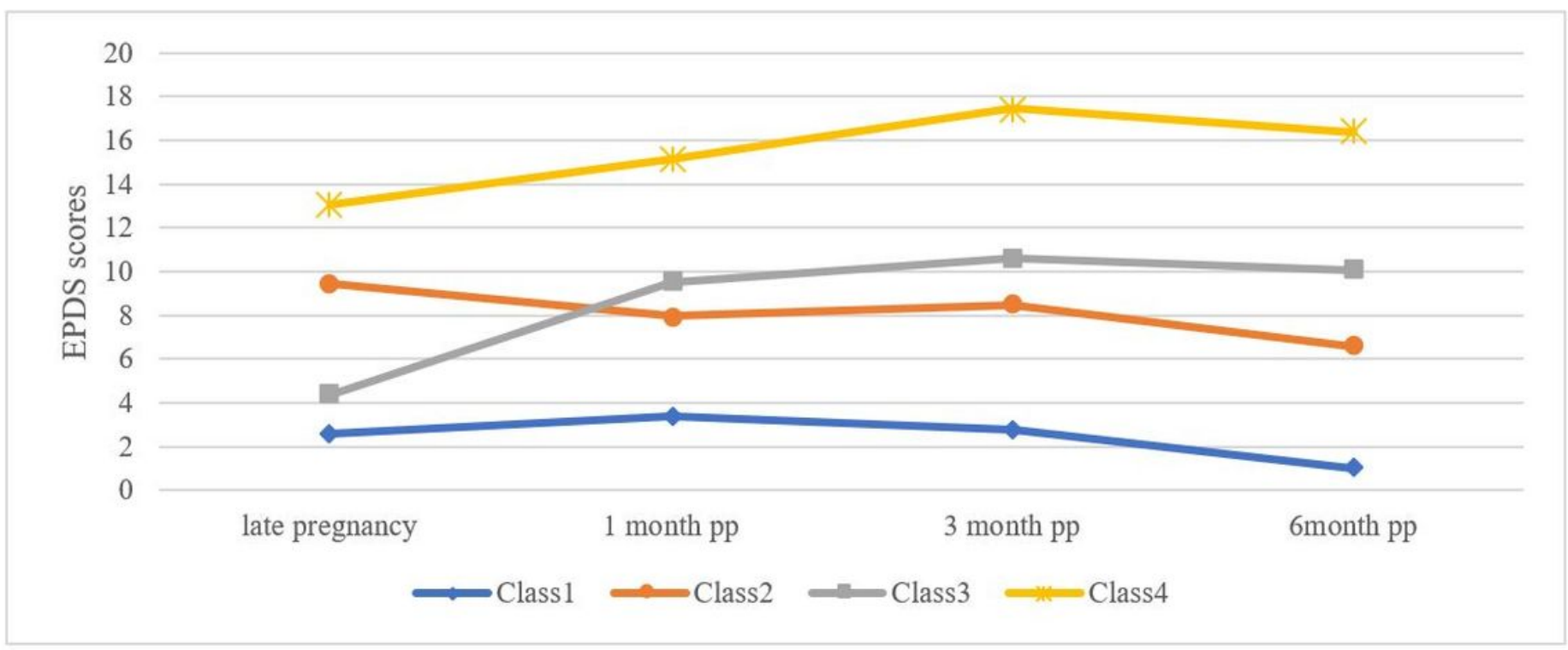

Figure 1

Estimated means of the EPDS for the four trajectories of maternal depressive symptoms from pregnancy to six months postpartum, and the 1-to 4-class trajectories of depressive symptoms were named orderly as "low depressive", "subclinical depressive", "moderate-low and increasing" and "persistent high

Page $11 / 12$ 
depressive".

\section{Supplementary Files}

This is a list of supplementary files associated with this preprint. Click to download.

- supplementaltable.docx 